\title{
Hidrocefalia aguda essencial
}

\author{
Hugo de Souza', Francisco Doutel', Carlos Augusto Borges', Ronaldo \\ Gonçalves de Azevedo', Walter Oliveira Junior ${ }^{2}$, Carlos Henrique Ribeiro ${ }^{3}$
}

Hospital Municipal Salgado Filho - Rio de Janeiro, RJ, Brasil

\section{RESUMO}

Objetivo: Apresentar discussão sobre o tema hidrocefalia aguda e a necessidade de se identificar os subtipos com a finalidade de proporcionar subsídios para a melhor compreensão e abordagem nessas situações. Considerações: A hidrocefalia é um distúrbio da dinâmica do líquido cefalorraquidiano em que há uma expansão do compartimento liquórico encefálico, notadamente do sistema ventricular. Por meio da revisão de literatura, evidencia-se uma extensa classificação com base em parâmetros diversos. Assim, é possível se definir hidrocefalia congênita e adquirida; obstrutiva e por superprodução de liquor; interna e externa; de alta pressão e de pressão normal; aguda, subaguda e crônica; sintomática e assintomática; comunicante e não-comunicante; mono, bi, tri e tetraventricular; "ex vacuo" e "arrested hydrocephalus". Neste contexto complexo e diversificado, a hidrocefalia aguda tem seu diagnóstico freqüentemente estabelecido com base no tempo de evolução da síndrome de hipertensão intracraniana, que normalmente a acompanha. Entretanto, como a ventriculomegalia não é um fenômeno passivamente determinado pela hipertensão liquórica intraventricular, ocorrem casos de dissociação entre o grau de dilatação ventricular e o grau de hipertensão intracraniana. Melhor seria, então, considerar como fator diagnóstico a presença de lesão obstrutiva indiscutivelmente aguda causadora da hidrocefalia. Porém, ainda assim, julgamos necessário distinguir-se um subtipo que exige uma abordagem direta independentemente da conduta adotada para a lesão expansiva inicial, a que denominamos hidrocefalia aguda essencial, em oposição ao subtipo hidrocefalia aguda não-essencial, cuja resolução é dependente da resolução da lesão expansiva que lhe deu origem. Conclusão: O conceito de hidrocefalia aguda essencial é operacional e de apurada precisão técnica, capaz de proporcionar subsídios à melhor abordagem da hidrocefalia aguda. Entretanto, ainda é necessária uma melhor compreensão do papel desempenhado pelos compartimentos estruturais encefálicos, pela ventriculomegalia e pelo hemoventrículo na fisiopatogenia das hidrocefalias em geral e das hidrocefalias agudas em particular.

\section{PALAVRAS-CHAVE}

Hidrocefalia. Classificação.

\section{ABSTRACT}

Acute essential hydrocephalus

Objective: To discuss the acute hydrocephalus and the necessity of identifying its subtypes to provide more subsidies for understanding and management of the patients in this condition. Background: Hydrocephalus is a disturbance in the CSF dynamics in which there is an expansion of CSF brain compartment especially of ventricular system. It becomes evident by literature revision that there is an extensive classification based on various parameters. Therefore, it is possible to determine many categories of hydrocephalus: congenital and acquired; obstructive and by overproduction of CSF; internal and external; high pressure and normal pressure; acute, subacute and chronic; symptomatic and asymptomatic; communicant and noncommunicant; mono/bi/tri/tetraventricular; ex vacuo and arrested hydrocephalus. In this complex and variable context, the diagnosis of acute hydrocephalus usually rely on the time of evolution of the intracranial hypertension syndrome that often occurs. However it would be better to consider as a diagnostic factor the presence of unquestionable acute obstructive lesion causative of the hydrocephalus. Even so, we distinguish a subtype that we have named essential acute hydrocephalus, which requires a direct approach to the hydrocephalus independently of the treatment adopted for the initial causative lesion, contrasting with a non-essential acute hydrocephalus subtype, whose resolution is dependent of the resolution of the initial expansive lesion that caused it. Conclusion: The essential acute hydrocephalus concept is operational and technically precise, allowing a better approach to the cases of acute hydrocephalus. However, it is still necessary a full understanding about the role played by the brain structural compartments, ventriculomegaly and intraventricular hemorrhage to the physiopathology of hydrocephalus in general, and acute hydrocephalus in particular.

\section{KEY WORDS}

Hydrocephalus. Classification.

1 Neurocirurgião do Hospital Municipal Salgado Filho.

2 Médico-residente de Neurocirurgia do Hospital Municipal Salgado Filho.

3 Chefe do Serviço de Neurocirurgia do Hospital Municipal Salgado Filho. 


\section{Introdução}

A hidrocefalia é um distúrbio da dinâmica do líquido cefalorraquidiano que determina uma expansão do compartimento liquórico encefálico, notadamente do sistema ventricular. Por meio da revisão de literatura, evidencia-se uma extensa classificação com base em parâmetros diversos ${ }^{3,4,14}$ :

a) quanto ao momento de instalação do processo hidrocefálico:

- hidrocefalia congênita: desenvolvida antes do nascimento, em conseqüência de má-formação congênita do sistema nervoso central ou de neuroinfecção intra-uterina ${ }^{11}$;

- hidrocefalia adquirida: desenvolvida após o nascimento, em razão de outros fatores.

b) quanto ao mecanismo fisiopatogênico:

- hidrocefalia obstrutiva: provocada por obstrução das vias liquóricas;

- hidrocefalia por superprodução de liquor: típica dos papilomas de plexo coróide.

c) quanto à localização da obstrução:

- hidrocefalia interna: a obstrução (intrínseca ou extrínseca) atinge principalmente o sistema ventricular;

- hidrocefalia externa: o acúmulo de liquor primariamente se dá no espaço subaracnóideo intracraniano, normalmente por obstrução ao nível das granulações aracnóideas.

d) quanto à associação com hipertensão intracraniana:

- hidrocefalia de alta pressão;

- hidrocefalia de pressão normal.

e) quanto ao tempo de evolução:

- hidrocefalia aguda: com evolução de até três dias;

- hidrocefalia subaguda: com evolução entre quatro e 28 dias;

- hidrocefalia crônica: com evolução superior a 28 dias.

f) quanto à presença de sintomatologia relacionada com a hidrocefalia:

- hidrocefalia sintomática;

- hidrocefalia assintomática.

g) quanto à presença de comunicação entre o sistema ventricular e o espaço subaracnóideo espinhal:

- hidrocefalia comunicante;

- hidrocefalia não-comunicante.

h) quanto ao número de ventrículos encefálicos dilatados:

- hidrocefalia monoventricular;

- hidrocefalia biventricular;

- hidrocefalia triventricular;

- hidrocefalia tetraventricular. i) hidrocefalia ex vacuo: que melhor seria denominada "ventriculomegalia ex vacuo", porque, nessa condição, não há propriamente um distúrbio da dinâmica liquórica, mas simples dilatação ventricular secundária à atrofia cerebral focal ou generalizada.

j) arrested hydrocephalus ou hidrocefalia suprimida, podendo ser:

- shunt-dependente: criança com crescimento normal do perímetro cefálico, mantendo ventrículos encefálicos normais ou levemente dilatados apenas quando o shunt está funcionante ${ }^{14}$;

- shunt-independente: criança com mais de 1 ano de idade, com shunt não-funcionante, porém sem quadro de descompensação, com crescimento normal do perímetro cefálico, mantendo ventrículos encefálicos normais ou levemente dilatados $^{14}$.

Essas classificações são superponíveis, de modo que, por exemplo, a hidrocefalia de pressão normal é usualmente crônica e comunicante, enquanto a hidrocefalia aguda é normalmente obstrutiva, de alta pressão e não-comunicante.

É necessário se observar uma rigorosa precisão conceitual, não só para que se possam distinguir claramente todos esses tipos entre si como também para se estabelecer o diagnóstico diferencial com outras alterações aparentemente correlacionadas ${ }^{17}$. Assim, por exemplo, a hidrocefalia externa não se confunde com o higroma subdural ${ }^{9}$ (no qual o acúmulo de liquor se dá no espaço subdural e não no espaço subaracnóideo) nem com a simples dilatação dos sulcos cerebrais secundária à atrofia cerebral (uma vez que, aqui, não há distúrbio da dinâmica liquórica).

\section{Hidrocefalia aguda}

O conceito de hidrocefalia aguda não é, portanto, um conceito isolado, mas, ao contrário, se insere em um contexto amplo e diversificado, representando apenas um dos diferentes modos de se analisar a hidrocefalia ${ }^{17}$.

O próprio diagnóstico de hidrocefalia aguda envolve aspectos muito importantes. Normalmente, todo o processo propedêutico se inicia pelo diagnóstico clínico da síndrome de hipertensão intracraniana, e é a tomografia computadorizada (TC) de crânio, realizada como investigação dessa síndrome, que irá permitir o diagnóstico de hidrocefalia de alta pressão, inferindo-se, 
então, o diagnóstico de hidrocefalia aguda, geralmente a partir do tempo de evolução da síndrome de hipertensão intracraniana ${ }^{17}$.

Entretanto, nem sempre o tempo de evolução da síndrome de hipertensão intracraniana representa fielmente o tempo de evolução da hidrocefalia, como no caso apresentado na figura 1 de uma paciente de 49 anos de idade admitida no Serviço de Emergência de nosso hospital com relato de cefaléia, vômitos e alteração da consciência havia 24 horas, e que se apresentava muito sonolenta e esboçando decorticação à direita. A hidrocefalia triventricular foi tratada com a instalação de uma derivação ventricular externa (DVE), observando-se saída de liquor xantocrômico, sob pressão, negativo à cultura. Certamente, essa hidrocefalia teria não só mais de 24 horas como muito provavelmente mais do que três dias de evolução.

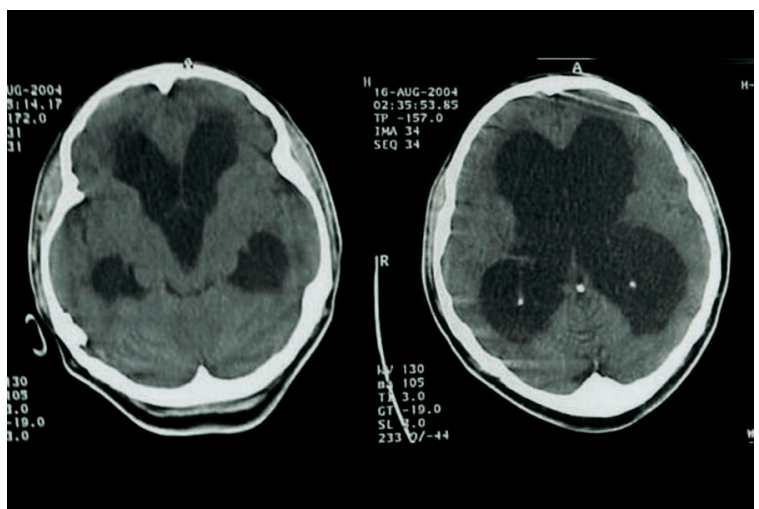

Figura 1 - Hidrocefalia de alta pressão associada com síndrome de hipertensão intracraniana com 24 horas de evolução.

Em vez do tempo de evolução da síndrome de hipertensão intracraniana, o parâmetro mais fidedigno para o diagnóstico de hidrocefalia aguda é a presença de uma lesão expansiva, indiscutivelmente aguda, como causa da hidrocefalia ${ }^{17}$, como no caso documentado na figura 2 de um paciente de 72 anos de idade admitido com quadro de coma de instalação súbita, cuja TC de crânio mostrou hematoma talâmico esquerdo com hemoventrículo maciço e hidrocefalia inquestionavelmente aguda, tratada também com a instalação de uma DVE.

Entretanto, mesmo quando se considera a lesão expansiva aguda inicial, há que se distinguir entre dois subtipos de hidrocefalia aguda ${ }^{17}$. Um subtipo em que a hidrocefalia aguda exige uma abordagem direta independentemente da conduta adotada para a lesão aguda inicial, como no caso acima citado, ao qual denominamos hidrocefalia aguda essencial, e um outro subtipo cuja resolução depende do tratamento da lesão expansiva inicial que lhe deu origem, por nós denominado hidrocefalia aguda não-essencial, exemplificada na figura 3 pelo caso de um paciente de 45 anos de idade, cuja hidrocefalia aguda era provocada por um volumoso hematoma subdural agudo ${ }^{17}$.

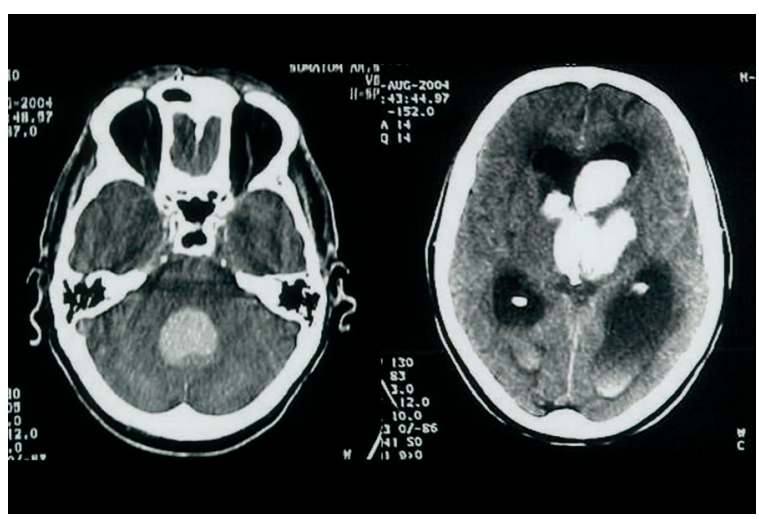

Figura 2 - Hidrocefalia aguda essencial.

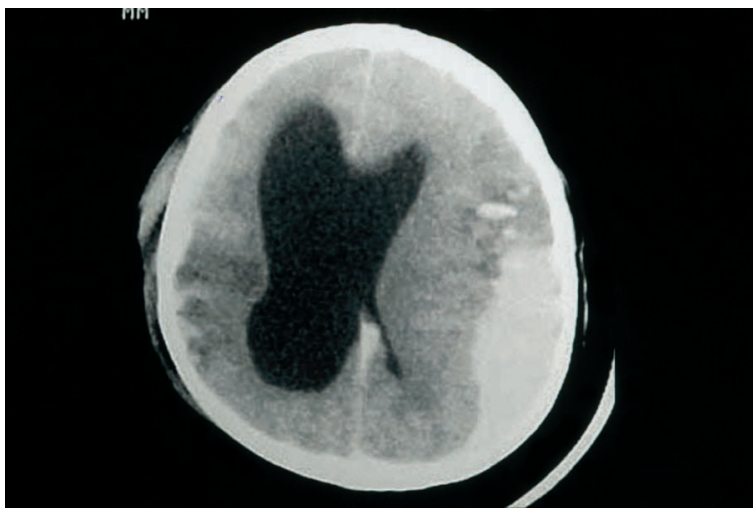

Figura 3 -Hidrocefalia aguda não-essencial.

\section{Hidrocefalia aguda de alta pressão}

Como a hidrocefalia aguda está intimamente associada com hipertensão intracraniana ${ }^{5}$, normalmente se tende a avaliar o grau de hipertensão intracraniana pelo grau de dilatação ventricular ${ }^{17}$. Porém, nem sempre essa dilatação ventricular se relaciona diretamente com a hipertensão intracraniana. Pode haver dilatação ventricular sem hipertensão intracraniana, como no caso de pacientes sintomáticos com dilatação ventricular à TC de crânio e disfunção do sistema de derivação liquórica constatada cirurgicamente, porém sem hipertensão intracraniana à mensuração pré-operatória ${ }^{15}$. 
Inversamente, pode haver hipertensão intracraniana sem dilatação ventricular, como no caso de pacientes sintomáticos com obstrução do sistema de derivação ventriculoperitoneal (DVP) verificada durante revisão cirúrgica e que não apresentavam dilatação ventricular à TC pré-operatória ${ }^{7}$.

Nas dilatações ventriculares não-agudas, essa dissociação é mais reconhecida como, por exemplo, relatado em pacientes com anorexia nervosa e doença de Cushing ${ }^{1}$ ou, ainda, em pacientes com hidrocefalia de pressão normal submetidos à DVP e que tenham boa recuperação neurológica, mesmo com persistência da ventriculomegalia ${ }^{10}$.

A dissociação entre o grau de hipertensão intracraniana e o grau de dilatação ventricular está exemplificada pelo caso mostrado na figura 4 de uma paciente de 55 anos de idade presumíveis, de população de rua, que foi trazida pelo Corpo de Bombeiros com relato de atropelamento; apresentava escore 6 da escala de Glasgow e sem sinais focais, e a TC evidenciou não só hidrocefalia triventricular como também extensa coleção subdural hipodensa de fossa posterior, aparentemente não-hipertensiva.

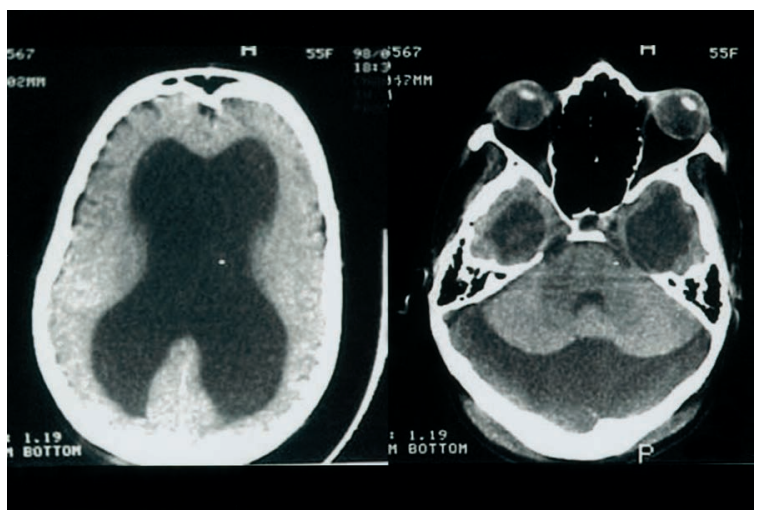

Figura 4 - Ventriculomegalia e higroma subdural de fossa posterior não-hipertensivos em paciente com comprometimento neurológico agudo de natureza traumática.

Como a TC de crânio não evidenciou nenhuma lesão intracraniana traumática que justificasse o comprometimento neurológico aparentemente agudo da paciente, optamos pela abordagem de ambas as lesões por meio de uma craniectomia suboccipital mediana e DVE a partir de uma trepanação parietal posterior direita. Em ambas as abordagens, o liquor se mostrou cristalino, incolor e não-hipertensivo. A paciente passou, então, a manter-se desperta, porém abúlica, sem alteração da ventriculomegalia (a despeito do funcionamento satisfatório da DVE), vindo a falecer posteriormente em razão de graves complicações infecciosas secundárias ao extenso desenluvamento do antebraço e mão direitos.
A razão de a dilatação ventricular nem sempre refletir fielmente a hipertensão intracraniana deve-se ao fato de a ventriculomegalia não ser apenas o resultado de um desequilíbrio entre a produção e a absorção de liquor ${ }^{16}$ ou um fenômeno passivamente determinado pela hipertensão liquórica intraventricular ${ }^{17}$. Ao contrário, seu mecanismo fisiopatogênico é mais complexo, envolvendo os três compartimentos estruturais encefálicos ${ }^{16}$ :

1. Compartimento parenquimatoso-constituído por neurônios, células gliais e fluido intersticial;

2. Compartimento vascular - único compartimento em comunicação com o "exterior", através das artérias carótidas e vertebrais e das veias jugulares;

3. Compartimento liquórico - compreendendo o sistema ventricular (com o epêndima e o plexo coróide) e o espaço subaracnóideo intracraniano (com as granulações aracnóideas, que se abrem no seio sagital superior), estando o liquor em equilíbrio com o fluido intersticial.

À medida que a pressão intraventricular se eleva, o liquor é forçado contra o epêndima, havendo deslocamento de líquido para o fluido intersticial ${ }^{4}$. Parte desse líquido é absorvida na extremidade venosa da circulação capilar e parte se dirige aos espaços perivasculares de Virchow-Robin e, daí, para o espaço subaracnóideo até as granulações aracnóideas, de onde é lançada ao seio sagital superior ${ }^{4}$. Com a progressão da dilatação ventricular, o deslocamento do líquido supera a sua remoção, e essa expansão do fluido intersticial passa a constituir, então, o edema transependimário, que se distingue do edema vasogênico pelo fato de ser produzido pela quebra da barreira liquor-encefálica, e não pelo aumento da permeabilidade da barreira hematoencefálica ${ }^{4,8}$.

A dilatação ventricular e o edema transependimário acarretam, respectivamente, a expansão do compartimento liquórico e do compartimento parenquimatoso. Como, de acordo com a doutrina de Monro-Kellie ${ }^{2}$, o volume total dos três compartimentos encefálicos deve se manter aproximadamente constante, porque a cavidade craniana possui um continente praticamente rígido, a expansão dos compartimentos liquórico e parenquimatoso deve ser acompanhada pela retração do compartimento vascular, o que se dá pela redução do fluxo sangüíneo encefálico 5 .

Essa condição hipóxico-isquêmica é mais acentuada na substância branca periventricular e atinge particularmente os oligodendrócitos, acarretando desmielinização e lesões axonais ${ }^{5}$. Como as áreas de edema transependimário são áreas de estagnação do fluido intersticial, o acúmulo de substâncias potencialmente tóxicas, que deveriam ser prontamente removidas, agrava ainda mais as lesões hipóxico-isquêmicas periventriculares ${ }^{5}$. 
Evoluindo ainda mais a expansão do compartimento liquórico, é o compartimento parenquimatoso que passa a ser retraído, à custa do fluido intersticial, com resolução do edema transependimário ${ }^{5}$. A maior ou menor facilidade com que o parênquima cederá espaço para os ventrículos em expansão será determinada pela viscoelasticidade cerebral, a qual, por sua vez, depende do gradiente entre a pressão liquórica intraventricular e a pressão venosa cerebral, gradiente este denominado pressão liquórica efetiva ${ }^{6,13}$.

A pressão liquórica efetiva normal é menor do que o limite bioelástico do parênquima cerebral, produzindo, por isso, uma tensão apenas suficiente para manter os ventrículos encefálicos patentes ${ }^{13}$. Entretanto, quando ela aumenta em função da elevação da pressão liquórica intraventricular, o limite bioelástico do parênquima é ultrapassado, os ventrículos se dilatam, e o parênquima passa a ser comprimido, especialmente em suas regiões periventriculares ${ }^{13}$.

O epêndima, cujas células haviam se tornado achatadas já ao tempo da formação do edema transependimário, torna-se fragmentado e começa a se degenerar ${ }^{5,16}$. As lesões axonais e da bainha de mielina, assim como as lesões celulares oligodendrogliais e ependimárias, estimulam a proliferação de astrócitos e de células microgliais, provocando intensa atividade fagocitária local, a qual é seguida pela formação de gliose reacional, que passa, então, a formar a parede ventricular, em substituição ao epêndima ${ }^{5,16}$.

Portanto, quanto maior a dilatação ventricular, maior será a deformação sofrida pelo parênquima e, assim, mais difícil será para o cérebro reassumir a forma original após a resolução da hipertensão liquórica intraventricular, podendo mesmo ocorrer uma deformação cerebral permanente ${ }^{6}$.

\section{Hidrocefalia aguda essencial}

Entendemos que a conjugação de três fatores, abaixo relacionados, caracteriza um subtipo de hidrocefalia aguda, o qual denominamos hidrocefalia aguda essencial, em oposição ao subtipo hidrocefalia aguda não-essencial, cuja resolução é dependente da resolução da lesão aguda que a originou:

1. Obstrução das vias liquóricas por lesão expansiva aguda;

2. Hipertensão intracraniana por maior acúmulo de liquor no sistema ventricular;

3. Necessidade de abordagem direta da hidrocefalia aguda, independentemente da conduta para a lesão que lhe deu origem.

\section{Discussão}

O conceito de hidrocefalia aguda essencial, ora proposto, é operacional e cientificamente bem fundamentado. Trata-se de hidrocefalia porque a ventriculomegalia está realmente associada a um distúrbio da dinâmica liquórica, mais especificamente da circulação do liquor. Diz-se hidrocefalia aguda porque ela é provocada pela presença de uma lesão obstrutiva indiscutivelmente aguda. Por fim, é hidrocefalia aguda essencial porque a sua abordagem independe da conduta adotada para a lesão expansiva que lhe deu origem, em razão da possibilidade de a hidrocefalia assumir um curso próprio e, assim, contribuir ainda mais para a deterioração neurológica do paciente.

O fator fisiopatogênico responsável por essa deterioração seria a hipertensão intracraniana. Porém, para estimá-la, nem sempre o grau de dilatação ventricular é um fator confiável, uma vez que existem casos, como já visto, de dissociação entre o grau de ventriculomegalia e o grau de hipertensão intracraniana. O próprio grau de dilatação ventricular pode nem mesmo estar diretamente relacionado com a intensidade do comprometimento neurológico.

A alternativa seria, então, a utilização de índices que teoricamente possibilitassem a avaliação mais objetiva do volume ventricular e a melhor estimativa da hipertensão intracraniana subjacente ${ }^{17}$. Um desses índices é o índice ventrículo-craniano, utilizado por Gerard Mohr ${ }^{12}$, em 1983, em seu estudo sobre as hemorragias intraventriculares secundárias à ruptura de aneurismas intracranianos, e definido como a razão entre a distância entre os núcleos caudados e o diâmetro intracraniano que o contém (figura 5). Depois de apurar-se o valor de até 0,15 como sendo o estatisticamente normal, foram estabelecidas três faixas de valores, a saber: 0,15 a 0,$20 ; 0,21$ a 0,25 ; e acima de 0,25, progressivamente indicativos do melhor para o pior prognóstico ${ }^{12}$.

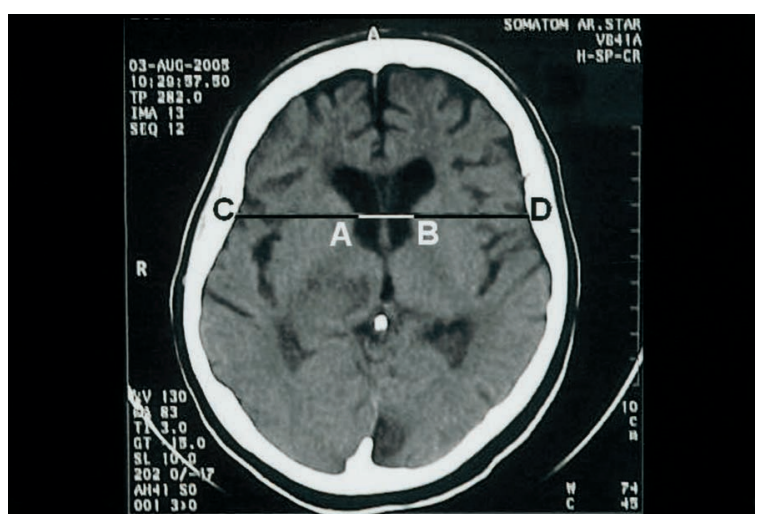

Figura 5 - Índice ventrículo-craniano $(M o h r)=A B / C D$. 
$\mathrm{Na}$ determinação do índice de Mohr, escolhemos preferencialmente o corte tomográfico paralelo à linha órbito-meatal que passa pela calcificação do corpo pineal, porque, geralmente, nesse nível, as faces mediais das cabeças dos núcleos caudados são paralelas, o que facilita a determinação do numerador do índice. As limitações desse procedimento são a necessidade de os cortes tomográficos serem paralelos à linha órbito-meatal (para que não ocorram deformações das imagens) e de que não haja distorção dos ventrículos supratentoriais pela própria lesão expansiva aguda, como está exemplificada pela figura 6 , a seguir.

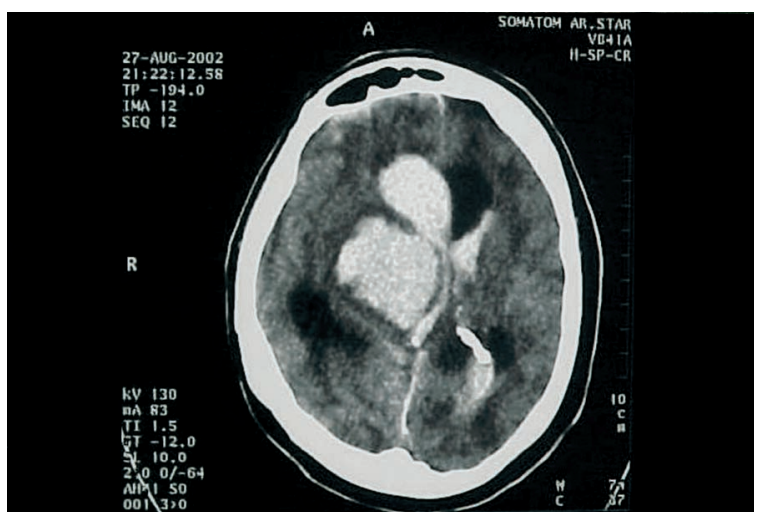

Figura 6 - Distorção do sistema ventricular por lesão expansiva aguda, impedindo a determinação do índice de Mohr.

Entretanto, $\mathrm{Mohr}^{12}$ concluiu que a hidrocefalia aguda possuía um valor prognóstico superior ao hemoventrículo, observação esta que, a nosso ver, merece uma maior reflexão, em razão de casos como o apresentado na figura 7, de um paciente de 35 anos de idade que foi trazido ao Serviço de Emergência de nosso hospital com quadro súbito de cefaléia, vômitos, crise convulsiva e coma, PA de 300/170 $\mathrm{mmHg}$, que evoluiu para o óbito após pouco menos de quatro horas de instalação do quadro clínico, muito mais, acreditamos, em função do hemoventrículo do que da hidrocefalia aguda.

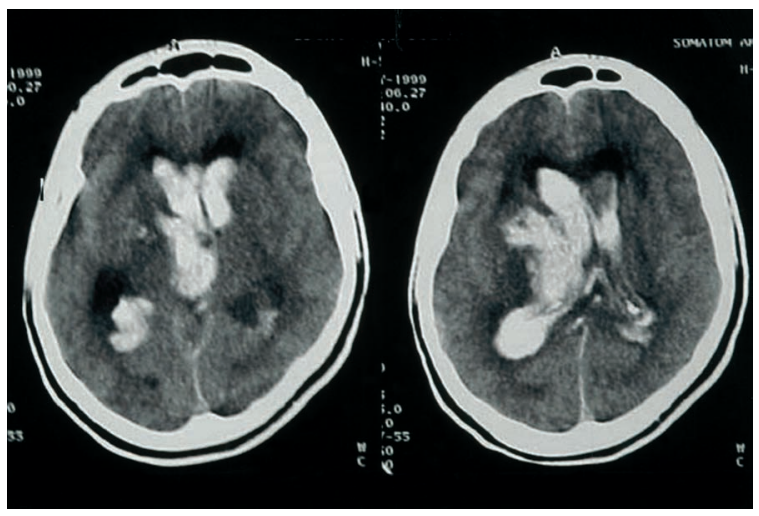

Figura 7 -Hemoventrículo versus hidrocefalia aguda como fatores prognósticos.
O peso específico a ser atribuído à hidrocefalia aguda e ao hemoventrículo como fatores prognósticos assume uma importância prática nos casos em que o hemoventrículo é maciço em um lado, e a hidrocefalia é predominante no outro, como o da figura 8 de uma paciente de 67 anos de idade admitida com quadro de coma de instalação aguda. Em princípio, a realização da DVE deve objetivar a correção da hidrocefalia aguda.

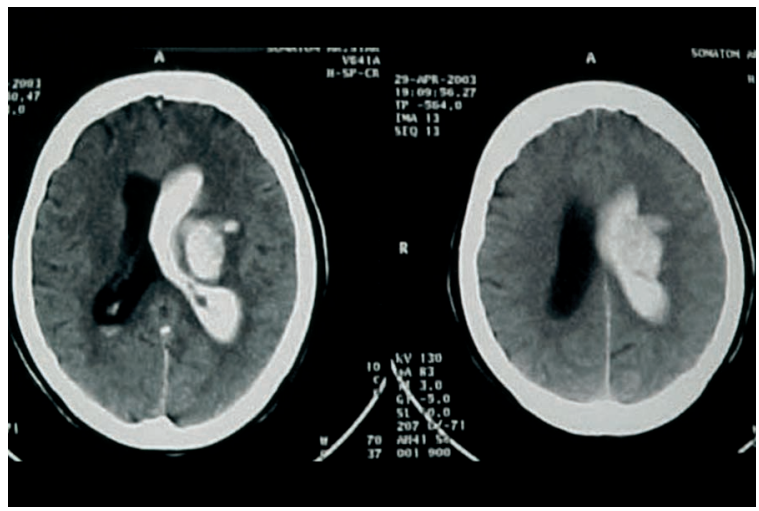

Figura 8 - Em princípio, a DVE se destina à correção da hidrocefalia aguda, e não do hemoventrículo.

\section{Conclusão}

O conceito de hidrocefalia aguda essencial é um conceito operacional e de apurada precisão científica, capaz de proporcionar subsídios para a melhor compreensão e abordagem das hidrocefalias agudas. Entretanto, muito ainda falta a conquistar, notadamente a compreensão plena do papel desempenhado pelos compartimentos estruturais encefálicos, pela ventriculomegalia e pelo hemoventrículo na fisiopatogenia das hidrocefalias em geral e das hidrocefalias agudas em particular. Nesse sentido, mostram-se bastante promissores os estudos iniciais do fluido intersticial cerebral com base na ressonância magnética, tanto em condições normais quanto em condições patológicas ${ }^{18}$.

\section{Agradecimentos}

Os autores agradecem à Dra. Tânia Saad, neuropediatra e doutora em Neurologia pela Universidade Federal do Rio de Janeiro (UFRJ), por sua valiosa colaboração na revisão da redação do Abstract. 


\section{Referências}

1. ADAMS RD, VICTOR M: Principles of Neurology. N York, McGraw-Hill Inc, 1993, p 549.

2. BERGSNEIDER M: Evolving concepts of cerebrospinal fluid. Neurosurg Clin North Am 36:635, 2001.

3. BLACK PML: Hydrocephalus in adults. In Youmans JR (ed): Neurological Surgery. Ed 4. CD-ROM. Provo (UT), Folio Co, 1994, vol 2, cap 37

4. BRADLEY Jr WG: Diagnostic tools in hydrocephalus. Neurosurg Clin North Am 36: 661-8, 2001.

5. DEL BIGIO MR: Pathophysiologic consequences of hydrocephalus. Neurosurg Clin North Am 36:639-45, 2001.

6. HAKIM CA, HAKIM R, HAKIM S: Normal-pressure hydrocephalus. Neurosurg Clin North Am 36:761-6, 2001.

7. KIM KAR, GANDHI C, HOHUAN D, KRIEGER M, McCOMB JG: Lack of ventricular response on head CT in pediatric shunted patients with surgery for proven hydrocephalus. AANS.org: 2004 May 3 (abstr). Disponível em URL: http:// www.aans.org/Library/Article.aspx?ArticleID=19837.

8. MACHADO ABM: Neuroanatomia Funcional. Rio de Janeiro, Atheneu, 1977, pp 76-9.

9. MATSUMOTO S, TAMAKI N: Subdural hydromas. Adv Neurotraumatol 1:157-8, 1986.

10. MEIER U: Does the ventricle size change after shunt operation for normal pressure hydrocephalus? AANS.org: 2004 May 3 (abstr). Disponível em URL: http://www.aans. org/Library/Article.aspx?ArticlelD=19829.

11. MERRIT HH: A Textbook of Neurology. Philadelphia, Lea \& Febiger, 1973, p 408.

12. MOHR G, FERGUSON G, KHAN M, MALLOY D, WATTS R, BENOIT B, WEIR B: Intraventricular hemorrhage from ruptured aneurysm. J Neurosurg 58:482-7, 1983.
13. OJEMANN RG, BLACK PM: Hydrocephalus in adults. In Youmans JR (ed): Neurological Surgery. Ed 2. Philadelphia, WB Saunders, 1982, vol 3, cap 37, pp 1423-4, 1433-4.

14. O'BRIEN MS: Hydrocephalus in children. In Youmans JR (ed): Neurological Surgery. Ed 2. Philadelphia, WB Saunders, 1982, vol 3, cap 36, pp 1382, 1419-20.

15. PANG D, ALTSCHULER E: Low-pressure and viscoelastic alterations in the brain. Neurosurgery 35:643-56, 1994.

16. SAINTE-ROSE C: Hydrocephalus in childhood. In Youmans JR (ed): Neurological Surgery. Ed 4. CD-ROM. Provo (UT), Folio Co, 1994, vol 2, cap 36.

17. SOUZA H, DOUTEL F, BORGES CA, AZEVEDO RG, OLIVEIRA Jr W, RIBEIRO CH: A importância do Índice de Mohr no tratamento das hidrocefalias agudas. Tema livre 528 apresentado no XXV Congresso Brasileiro de Neurocirurgia, 2004, Goiânia, GO, Brasil.

18. YAMADA S, MATSUDA T, HORII M, SUGIYAMA Y, BLUML S, McCOMB JG: Intracellular and extracellular water imaging of the brain using magnetic resonance imaging. AANS. org: 2003 Dec 2 (abstr). Disponível em URL: http://www.aans. org/Library/Article.aspx?ArticleID=10843

Original recebido em julho de 2006

Aceito para publicação em abril de 2007

\section{Endereço para correspondência}

Hugo de Souza

Rua Arquias Cordeiro, 370 - $7^{\circ}$ andar

20770-000 - Rio de Janeiro, RJ

E-mail:hugosou@walla.com 